\title{
Úng dụng mô hình hồi quy không gian trong phân tích tăng trưởng doanh thu du lịch của các tỉnh/thành tại Việt Nam
}

\section{An application of the spatial regression model for analyzing tourism receipt growth of provinces/cities in Vietnam}

\author{
Nguyễn Văn Sĩ ${ }^{1}$, Nguyễn Viết Bằng ${ }^{2 *}$ \\ ${ }^{1,2}$ Trường Đại học Kinh tế Thành phố Hồ Chí Minh, Việt Nam \\ *Tác giả liên hệ, Email: bangnv@ueh.edu.vn
}

\section{THÔNG TIN}

DOI: $10.46223 / \mathrm{HCMCOUJS.}$ econ.vi.15.2.236.2020

Ngày nhận: 28/02/2020

Ngày nhận lại: 22/03/2020

Duyệt đăng: 27/03/2020

Từ khóa:

doanh thu du lịch, hồi quy không gian, tăng trưởng du lịch

\section{TÓM TẮT}

Mục đích của nghiên cứu là xác định và đo lường các yếu tố tác động đến doanh thu du lịch của các tỉnh/thành tại Việt Nam thông qua ứng dụng mô hình hồi quy không gian. Đề tài sử dụng phương pháp nghiên cứu định tính kết hợp định lượng. Nghiên cứu định tính được thực hiện thông qua phỏng vấn sâu cùng 09 chuyên gia. Nghiên cứu định lượng được thực hiện thông qua ứng dụng mô hình kinh tế lượng không gian với bộ dữ liệu thứ cấp từ năm 2013 đến 2017 của các tỉnh/thành tại Việt Nam. Kết quả nghiên cứu cho thấy: doanh thu du lịch của mỗi địa phương chịu tác động bởi: lượng khách, dân số và khối lượng luân chuyển hành khách. Thêm vào đó, Kết quả nghiên cứu là một bằng chứng thực nghiệm cho thấy có sự tương quan giữa các tỉnh gần nhau trong tăng trưởng doanh thu du lịch, điều này có nghĩa là tăng trưởng du lịch của địa phương này có tác động đến tăng trưởng doanh thu của các tỉnh lân cận. Đồng thời, lượng khách, dân số và khối lượng luân chuyển của hành khách có sự tương tác về mặt không gian giữa các tỉnh/thành lân cận.

\begin{abstract}
The paper defines and measures key factors regarding to tourism receipt of provinces/cities in Vietnam through spatial regression model. This study has used qualitative and quantitative researches: (i) qualitative carried out through deep interview with 09 experts, and (ii) quantitative research conducted through spatial regression model with secondary data from 2013 to 2017 of Vietnam's provinces/Cities. The result shows that: tourism receipt is directly affected by tourist arrivals, population, the volume of tourist movement. Moreover, the result of the study is empirical evidence that there is a correlation between neighboring provinces/cities in tourism receipt growth of provinces/cities, it means that tourism receipt growth of this province has an impact
\end{abstract}


Keywords:

tourism receipt, spatial regression, tourism growth on tourism receipt growth of other neighboring provinces/cities. In addition, the result also shows that: tourist arrivals, population, and the volume of tourist movement have spatial interaction between neighboring provinces.

\section{Giới thiệu}

Kinh tế Việt Nam kể từ sau khi đổi mới đã có nhiều thay đổi trong cơ cấu, đáng chú ý là sự chuyển dịch từ nông nghiệp sang công nghiệp và dịch vụ, tỷ trọng ngành dịch vụ đã có sự gia tăng đáng kể, trong đó ngành dịch vụ du lịch chiếm một trọng số không nhỏ và ngày càng lớn dần lên theo sự hội nhập của đất nước. Thực tế cho thấy Việt Nam với lợi thế về tiềm năng du lịch (nhiều cảnh quan thiên nhiên đẹp, bờ biển dài trải dọc đất nước, có nhiều khu du lịch quốc gia được chú trọng bảo tồn và phát triển, etc.) và là nước có nền kinh tế chính trị được cộng đồng thế giới đánh giá là ổn định. Việt Nam trở thành tâm điểm thu hút nhiều khách du lịch trong và ngoài nước.

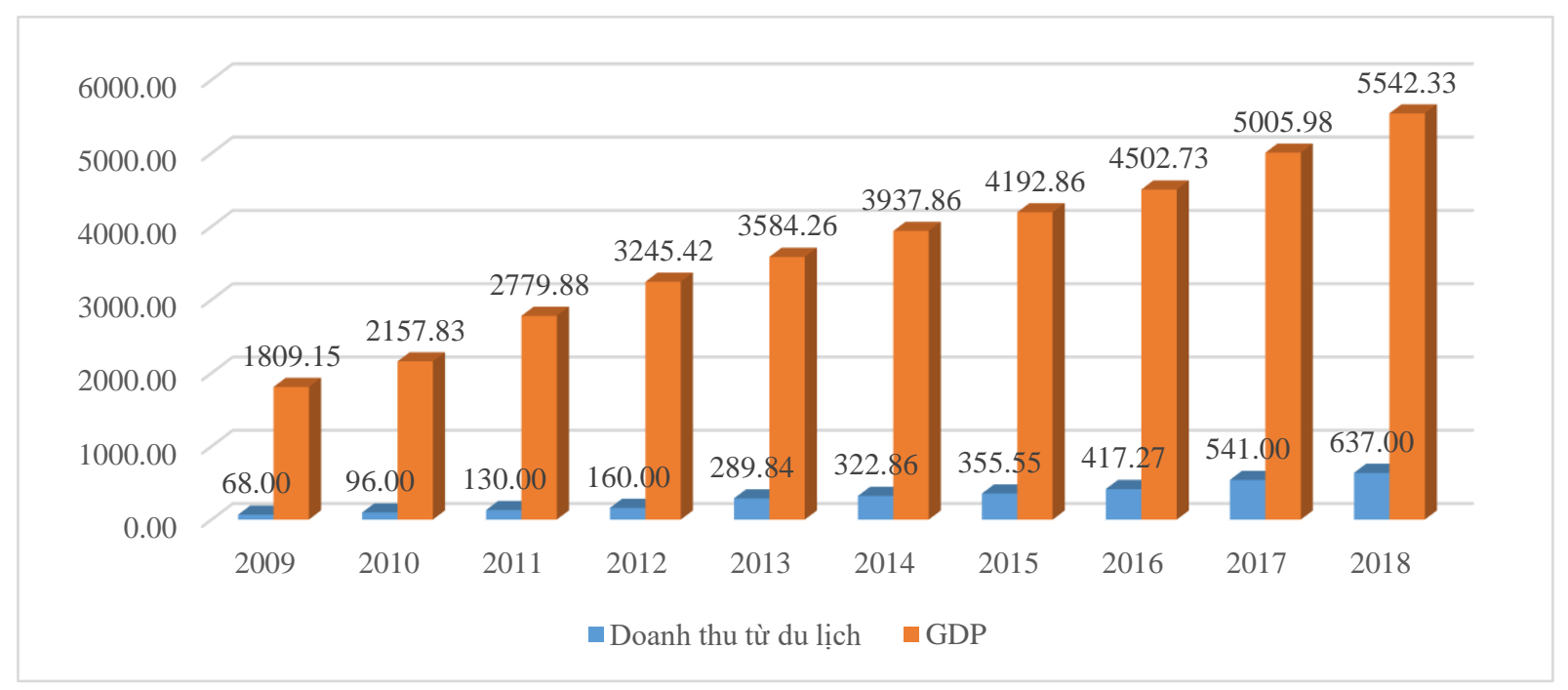

Đơn vị tính: Nghìn tỷ đồng

Hình 1. Thực trạng doanh thu từ du lịch

Nguồn: Tổng cục thống kê (2019) 


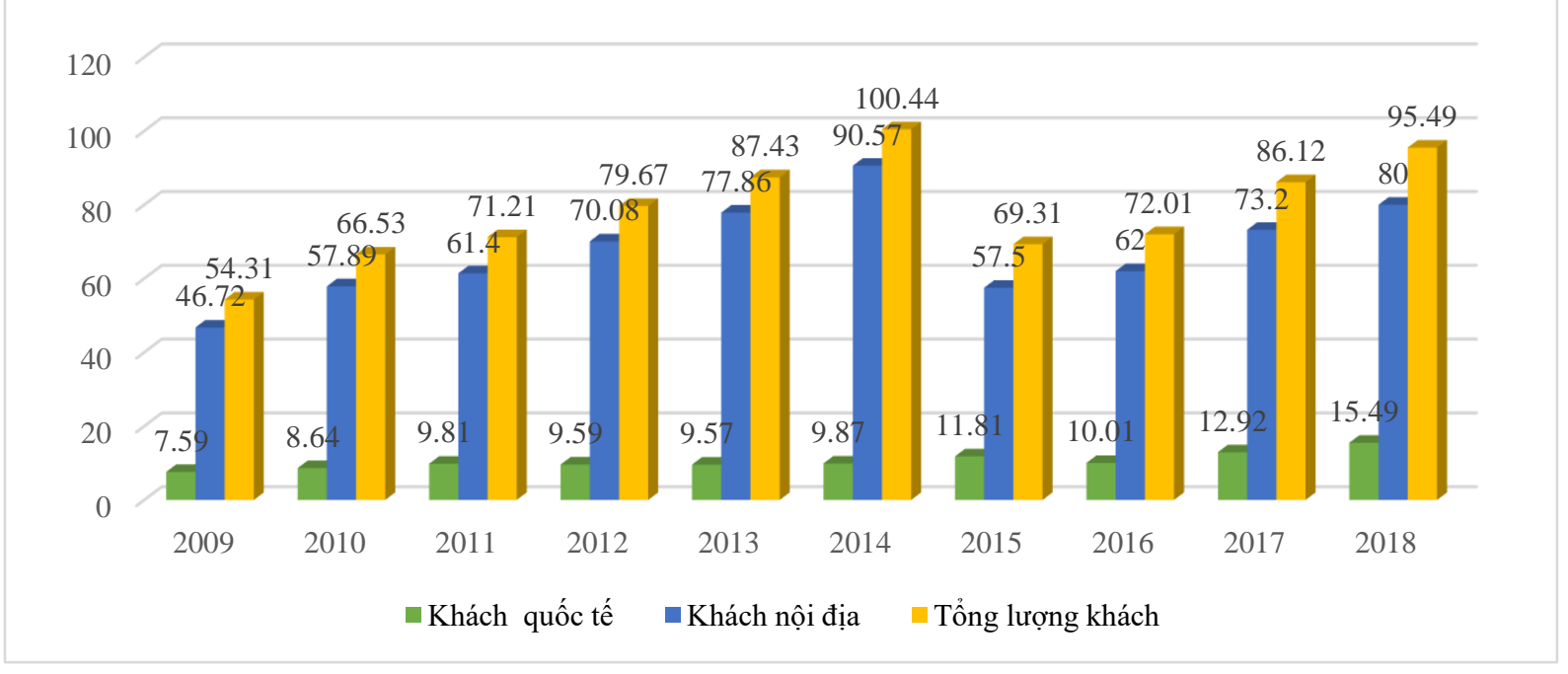

Đơn vị tính: Triệu lượt

Hình 2. Thực trạng khách du lịch tại Việt Nam

Nguồn: Tổng cục thống kê, 2019

Thống kê về du lịch tại Hình 1 và Hình 2 cho thấy: doanh thu và lượng khách du lịch đều tăng qua các năm. Nếu như năm 2009 chỉ có 54.31 triệu lượt đạt doanh số là 68 nghìn tỷ đồng thì đến hết năm 2018 con số thật ấn tượng với 95.49 triệu lượt đạt doanh thu là 637 nghìn tỷ đồng. Tuy nhiên hầu như lượng khách quay trở lại Việt Nam để du lịch thì lại rất thấp (Tổng cục du lịch, 2019) do sản phẩm du lịch không đa dạng, chưa có sự phối hợp giữa các địa phương trong hoạt động du lịch, etc. Mỗi địa phương có một thế mạnh về du lịch nhưng nhìn chung nhiều địa phương chưa chủ động và phối hợp trong việc tổ chức các sự kiện, sản phẩm du lịch để thu hút khách du lịch trong khi tồn tại mối tương quan giữa các địa phương trong việc thu hút du lịch.

Để phân tích mối quan hệ giữa các đơn vị không gian trong khu vực thì mô hình kinh tế lượng không gian được sử dụng kiểm định về tính phụ thuộc không gian trong các mô hình hồi quy (Anselin, 1988) vì một khi có sự phụ thuộc không gian giữa các địa phương thì kết quả ước lượng các mô hình kinh tế lượng bằng phương pháp OLS truyền thống thông thường bị chệch và không hiệu quả. Hơn nữa, Anselin (1988) cũng làm rõ nếu dữ liệu bảng được sử dụng và có sự hiện diện của các hiệu ứng không gian, thì các mô hình kinh tế lượng không gian có thể phân tích tính đa chiều của sự phụ thuộc này.

Trong bài viết này nhóm tác giả kế thừa các mô hình kinh tế lượng không gian với các ưu điểm của nó để ứng dụng nghiên cứu về doanh thu du lịch của Việt Nam tiếp cận ở góc độ tỉnh/thành.

\section{Cơ sở lý thuyết về hồi quy không gian - Tương quan không gian}

\subsection{Kiểm định Moran's I}

Các nghiên cứu trong thống kê không gian thường phân biệt giữa hai loại tác động không gian khác nhau: tương tác không gian (tự tương quan không gian) và cấu trúc không gian (tính không đồng nhất không gian). Việc kiểm tra các đặc điểm không gian của dữ liệu bị ảnh hưởng mạnh mẽ bởi các vị trí mà từ đó các quan sát được thực hiện. Các đơn vị địa lý lân cận 
ảnh hưởng đến từng vị trí khác và các vị trí gần nhau thường có sự tương tác không gian nhiều hơn các vị trí có khoảng cách xa hơn (Coughlin \& Segev, 2000).

Khi có sự phụ thuộc không gian giữa các đơn vị trong khu vực thì việc sử dụng một phương pháp phân tích sự tương quan không gian là cần thiết. Trong nghiên cứu này, nhóm tác giả sử dụng hệ số Moran's I để xác định mối tương quan không gian giữa các khu vực lân cận. Thống kê Moran's I có thể cho một kết quả về tự tương quan không gian của các quan sát một cách tổng quát. Mặt khác, đối với thống kê $\mathrm{G}^{*}$ xem xét cụ thể tự tương quan không gian cho từng quan sát (Ord \& Getis, 1995). Thống kê Moran's I phụ thuộc vào ma trận trọng số không gian phản ánh cường độ của mối quan hệ địa lý giữa các quan sát trong một khu vực (Anselin, 1988), và là một trong những phương pháp mà các nhà nghiên cứu thường sử dụng nhất để xem xét sự tương quan giữa các biến số (Elhorst, 2003). Giá trị thống kê Moran's I nằm trong khoảng [-1, 1].

Thống kê Moran's I của biến x được xác định như sau:

$$
I=\frac{n}{\sum_{i=1}^{n} \sum_{j=1}^{n} w_{i j}} \cdot \frac{\sum_{i=1}^{n} \sum_{j=1}^{n} w_{i j}\left(x_{i}-\bar{x}\right)\left(x_{j}-\bar{x}\right)}{\sum_{i=1}^{n}\left(x_{i}-\bar{x}\right)^{2}}
$$

Trong đó

$x_{i}$ là giá trị quan sát của đơn vị không gian thứ $\mathrm{i}$

$\bar{x}$ là giá trị trung bình của $\mathrm{x}$

$n$ là số giá trị quan sát của các đơn vị không gian

$w_{i j}$ là phần tử dòng $\mathrm{i}$, cột $\mathrm{j}$ của ma trận trọng số không gian $\mathrm{W}$ đã được chuẩn hóa

Nghiên cứu của Cliff và Ord (1981) cho thấy thống kê Moran's I có phân phối tiệm cận là phân phối chuẩn.

Thống kê $\mathrm{G}$, ban đầu được phát triển bởi Ord và Getis (1995) được sử dụng để nghiên cứu về mô hình không gian. Chúng đại diện cho một chỉ số tự tương quan không gian một cách tổng quát. Mặt khác thống kê $G_{i}^{*}$ như là một chỉ số tự tương quan không gian cục bộ và nó phù hợp hơn cho việc xem xét về cấu trúc cụm không gian. Thống kê $G_{i}^{*}$ được xác định như sau:

$$
G_{i}^{*}=\frac{\sum_{j=1}^{n} w_{i j} x_{j}-\bar{x} \sum_{j=1}^{n} w_{i j}}{S^{*} \sqrt{\frac{n \sum_{j=1}^{n} w_{i j}^{2}-\left(\sum_{j=1}^{n} w_{i j}\right)^{2}}{n-1}}}
$$

Các ký hiệu tương tự như trên và $\mathrm{S}^{*}$ xác định bởi

$$
S^{*}=\sqrt{\sum_{j=1}^{n} x_{j}^{2} / n-(\bar{x})^{2}}
$$


Thống kê $G_{i}^{*}$ là một Z-score. Một Z-score cao và p-value nhỏ cho một đặc điểm chỉ ra một điểm giá trị cao (hot spot) có ý nghĩa thống kê. Một Z-score âm thấp và p-value nhỏ chỉ ra một điểm giá trị thấp (cold spot) có ý nghĩa thống kê. Các Z-score càng cao hơn (hoặc thấp hơn) thì việc phân cụm càng mạnh. Một z-score gần 0 cho thấy không có cụm không gian rõ ràng.

Sẽ có 5 trường hợp có thể xảy ra khi áp dụng hệ số Morans's I và thống kê $G_{i}^{*}$, bao gồm tất cả các quan sát: (1) Không có sự tương quan không gian, (2) có tương quan cao - cao (H$\mathrm{H})$, (3) có tương quan thấp - thấp ( $\mathrm{L}-\mathrm{L}),(4)$ có tương quan cao - thấp $(\mathrm{H}-\mathrm{L})$, và $(5)$ có tương quan thấp - cao (L-H).

\subsection{Ma trận trọng số không gian}

Ma trận trọng số không gian có vai trò quan trọng trong phân tích kinh tế lượng không gian và kết hợp được sự phụ thuộc không gian vào mô hình nghiên cứu. Gọi n là số đơn vị không gian. Ma trận trọng số không gian, ký hiệu $\mathrm{W}$, là ma trận cấp $(\mathrm{n} \times \mathrm{n})$ đối xứng, dương với phần tử tại vị trí $\mathrm{i}, \mathrm{j}$ là $\mathrm{w}_{\mathrm{ij}}$. Giá trị $\mathrm{w}_{\mathrm{ij}}$ gọi là trọng số không gian cho từng cặp vị trí $\mathrm{i}, \mathrm{j}$ xác định mối tương quan giữa hai địa phương $\mathrm{i}, \mathrm{j}$. Và quy ước rằng các phần tử nằm trên đường chéo chính của ma trận trọng số bằng 0 , tức là $\mathrm{w}_{\mathrm{ij}}=0$.

$$
\text { Ký hiệu: } \quad W=\left(\begin{array}{cccc}
w_{11} & w_{12} & \ldots & w_{1 n} \\
w_{21} & w_{22} & \ldots & w_{2 n} \\
\vdots & \vdots & \ddots & \vdots \\
w_{n 1} & w_{n 2} & \ldots & w_{n n}
\end{array}\right)
$$

Trong nghiên cứu thực nghiệm ma trận trọng số không gian được xây dựng thường dựa trên khoảng cách địa lý hoặc sự tiếp giáp giữa các địa phương. Có thể nêu ra một số dạng ma trận trọng số sau:

(1) Ma trận trọng số không gian tiếp giáp bậc nhất: Coughlin và Segev (2000) cho rằng: một địa phương $\mathrm{i}$ có chung đường biên với địa phương $\mathrm{j}(\mathrm{i} \neq \mathrm{j})$ gọi là có tương quan không gian với nhau. Lúc đó, mỗi phần tử của ma trận trọng số không gian định bởi:

$$
w_{i j}=\left\{\begin{array}{lc}
1 & \mathrm{i}, \mathrm{j} \text { chung duong bien } \\
0 & \mathrm{i}, \mathrm{j} \text { khong chung duong bien }
\end{array}\right.
$$

(2) Ma trận trọng số không gian dựa trên khoảng cách: gọi d là khoảng cách ngưỡng thì là khoảng cách giữa hai địa phương $\mathrm{i}, \mathrm{j}$ (Anselin, 1988). Thì mỗi phần tử của $\mathrm{W}$ định bởi:

$$
w_{i j}=\left\{\begin{array}{c}
1,0 \leq d_{i i} \leq d \\
0, d>d_{i j}
\end{array}\right.
$$
định bởi:

(3) Ma trận trọng số không gian dựa trên khoảng cách nghịch đảo: mỗi phần tử của $\mathrm{W}$ 


$$
w_{i j}=\left\{\begin{array}{c}
\frac{1}{d_{i j}^{\alpha}}, \mathrm{i} \neq \mathrm{j} \\
0, \mathrm{i}=j
\end{array}\right.
$$

Với $\alpha=1$ hoặc $\alpha=2$

Trong thực nghiệm thường sử dụng ma trận trọng số không gian được chuẩn hóa theo dòng (Anselin, 1988), tức là mỗi phần tử của ma trận chuẩn hóa theo dòng có dạng:

$$
w_{i j}^{s}=\frac{w_{i j}}{\sum_{j} w_{i j}}
$$

Giá trị của các phần tử của ma trận luôn nằm trong khoảng $[0,1]$ và tổng của mỗi dòng ma trận chuẩn hóa theo dòng bằng 1 .

\subsection{Các mô hình kinh tế lựng không gian với dũ liệu bảng}

Việc phân tích dữ liệu bảng trong các mô hình kinh tế lượng không gian của các nhà nghiên cứu đang phát triển mạnh về mặt phương pháp luận. Một số nghiên cứu điển hình như: Elhorst (2003), Baltagi và Liu (2008). Trong đó, Elhorst (2003) đã đưa ra phân tích và đánh giá về các vấn đề phát sinh trong việc ước lượng các mô hình kinh tế lượng không gian với dữ liệu bảng. Trong nghiên cứu ứng dụng được mở rộng cần xem xét về tương quan sai số không gian hoặc biến phụ thuộc với độ trễ không gian bao gồm: mô hình hiệu ứng cố định, mô hình hiệu ứng ngẫu nhiên, mô hình hệ số cố định và mô hình hệ số ngẫu nhiên. Hiện nay có nhiều mô hình kinh tế lượng không gian được sử dụng. Theo Elhorst (2003), Chou, Chen, và Mai (2015) thì các mô hình cơ bản được sử dụng để ước lượng với dữ liệu bảng không gian là: mô hình độ trễ không gian (SLM-Spatial Lag Model), mô hình sai số không gian (SEM - Spatial Error Model) và mô hình Durbin không gian (SDM - Spatial Durbin Model). Tuy nhiên, theo Vega và Elhorst (2015) thì những mô hình này là trường hợp đặc biệt của mô hình không gian tổng quát (GNS-General Nesting Spatial) và đây là mô hình cho tất cả các loại hiệu ứng tương quan không gian. Mô hình (GNS) dữ liệu bảng tổng quát có dạng như sau:

$$
\text { (GNS) } \quad \begin{aligned}
Y & =\alpha l_{N}+\rho W Y+X \beta+W X \theta+\mu+v+u \\
u & =\lambda W u+\varepsilon
\end{aligned}
$$

- Khi: $\theta=0$, thì (GNS) trở thành mô hình (SAC) hay (SARAR) (Anselin, 1988)

$$
\begin{aligned}
Y & =\rho W Y+X \beta+\mu+v+u \\
\text { (SAC) } \quad u & =\lambda W u+\varepsilon
\end{aligned}
$$

- Khi $\lambda=0$, thì (GNS) trở thành:

$$
\text { (SDM) } \quad Y=\rho W Y+X \beta+W X \theta+\mu+v+\varepsilon
$$

- Khi $\theta=0$, thì (SDM) trở thành:

$$
\text { (SLM) } \quad Y=\rho W Y+X \beta+\mu+v+\varepsilon
$$

- Khi $\theta+\rho \beta=0$, thì được mô hình (SEM) được đề xuất bởi Anselin (1988) như sau: 
(SEM)

$$
\begin{gathered}
Y=X \beta+\mu+v+u \\
u=\lambda W u+\varepsilon \\
(\theta=-\rho \beta \rightarrow \lambda=\rho)
\end{gathered}
$$

Trong đó:

Y là vec tơ biến phụ thuộc $(\mathrm{Nx} 1)$ tại khu vực $\mathrm{i}(\mathrm{i}=1, \ldots, \mathrm{N})$; chỉ số thời gian $\mathrm{t}=1, \ldots, \mathrm{T}$

$\mathrm{X}$ là ma trận $(\mathrm{NxK})$ gồm các biến giải thích, $\mathrm{K}$ là số biến giải thích

$\rho$ là tham số tự tương quan không gian tương ứng với biến trễ không gian của biến phụ thuộc WY. Và $(\rho W Y)$ tương tác nội sinh

W là ma trận trọng số không gian cấp ( $\mathrm{NxN})$, mô tả mối liên hệ không gian giữa các đơn vị

$\theta$ chỉ hiệu ứng tương tác ngoại sinh của các khu vực lân cận của biến giải thích WX $(W X \theta)$ tương tác ngoại sinh

$\beta$ là tham số chưa biết của các biến độc lập và là ma trận $(\mathrm{Kx} 1)$.

$\lambda$ chỉ sự phụ thuộc không gian của các khu vực lân cận của các số hạng sai số $(\lambda W u)$ tương tác thông qua sai số

$\varepsilon$ ký hiệu vec tơ sai số có phân phối chuẩn, trung bình bằng 0 và độ lệch chuẩn $\sigma$

$\mu, v$ lần lượt là các hiệu ứng không gian và thời gian

\subsection{Tác động trục tiếp, tác động gián tiếp và tổng tác động}

Có một số mô hình cho các tác động như: Tác động trực tiếp, tác động gián tiếp và tổng tác động. Elhorst (2014) đã đưa ra yếu tố các tác động tổng quát như sau: Từ phương trình mô hình Durbin không gian được viết lại:

$$
y=(I-\rho W)^{-1}\left(\alpha l_{N}+X \beta+W X \theta\right)+(I-\rho W)^{-1} \varepsilon
$$

Lấy đạo hàm riêng phần của các giá trị kỳ vọng của $\mathrm{y}$ theo $\mathrm{k}$ biến giải thích của $\mathrm{X}$, với các quan sát $1, \ldots, \mathrm{N}$ được viết như sau:

$$
\left[\frac{\partial E(y)}{\partial x_{1 k}} \cdots \frac{\partial E(y)}{\partial x_{N k}}\right]=(I-\rho W)^{-1}\left[\begin{array}{cccc}
\beta_{k} & W_{12} \theta_{k} & \cdots & W_{1 n} \theta_{k} \\
W_{21} \theta_{k} & \beta_{k} & \cdots & W_{2 n} \theta_{k} \\
\cdots & \cdots & \cdots & \cdots \\
W_{n 1} \theta_{k} & W_{n 2} \theta_{k} & \cdots & \beta_{k}
\end{array}\right]
$$

Các phần tử nằm trên đường chéo chính của ma trận trên là các tác động trực tiếp, và tác động gián tiếp là các dòng hoặc các cột (ngoại trừ đường chéo chính). Theo LeSage và Pace (2009), Vega và Elhorst (2015) thì sự hiện diện của ma trận trọng số không gian làm cho các hiệu ứng biên trở nên phong phú và phức tạp hơn so với mô hình ước lượng OLS truyền thống. Trong các mô hình ước lượng bằng OLS và SEM thì tác động trực tiếp của một biến giải thích thứ $\mathrm{k}$ bằng với hệ số ước lượng biến giải thích thứ $\mathrm{k}$ là $\beta_{k}$, tác động gián tiếp bằng 0 . Trong $\mathrm{SDM}$, GNS tác động trực tiếp là các phần tử trên đường chéo của $(I-\rho W)^{-1}\left(\beta_{k}+W \theta_{k}\right)$, tác 
động gián tiếp gồm các phần tử nằm ngoài đường chéo của $(I-\rho W)^{-1}\left(\beta_{k}+W \theta_{k}\right)$. Để tính giá trị trên, hệ số nhân không gian được đề xuất một cách tính:

$$
(I-\rho W)^{-1}=I+\rho W+\rho^{2} W^{2}+\rho^{3} W^{3}+\ldots
$$

Tác động trực tiếp và tác động gián tiếp sẽ bao gồm cả tác động phản hồi (Feedback Effect) đến từ các đơn vị không gian lân cận. Tác động trực tiếp là tác động của biến giải thích từ quan sát i lên biến phụ thuộc của quan sát thứ i. Tác động gián tiếp là tác động của biến giải thích từ các quan sát lân cận khác quan sát thứ i lên biến phụ thuộc của quan sát i. Tổng tác động bao gồm tác đông trực tiếp và tác động gián tiếp.

\subsection{Kiểm định lựa chọn mô hình kinh tế lựng không gian}

Do sự tồn tại của mối tương quan không gian trong các biến của mô hình, phương pháp bình phương nhỏ nhất OLS ước lượng tham số có thể dẫn đến tính chệch và không nhất quán. Do đó, phương pháp ML (maximum likelihood) đã được sử dụng ước lượng trong các mô hình kinh tế lượng không gian bởi Anselin (1988). Đối với việc lựa chọn mô hình kinh tế lượng không gian, nhiều tài liệu về kinh tế lượng không gian gợi ý hai cách tiếp cận: (i) cách tiếp cận cụ thể đến tổng quát; và (ii) cách tiếp cận tổng quát đến cụ thể (Florax, Folmer, \& Rey, 2006; Mur \& Angulo, 2009). Elhorst (2014) đề xuất quy trình kiểm định hỗn hợp cả hai cách tiếp cận. Trước tiên, mô hình phi không gian được ước lượng để kiểm định xem liệu mô hình phi không gian hoặc là mô hình kinh tế lượng không gian (SLM hoặc SEM) là phù hợp, bằng cách sử dụng kiểm định LM (Lagrange Multiplier) trên dữ liệu (cách tiếp cận cụ thể đến tổng quát). Trong trường hợp mô hình phi không gian bị bác bỏ, SDM được ước lượng để kiểm định xem nó có thể đơn giản hóa thành SLM hoặc SEM (phương pháp tổng quát đến cụ thể). Để thực hiện điều này, thì các giả thiết cần kiểm định là $H_{0}: \theta=0$ và $H_{0}: \theta+\rho \beta=0$, các phương pháp kiểm định thường được sử dụng là thống kê kiểm định LR (Likelihood Ratio) hoặc thống kê kiểm định Wald. Thông qua kiểm định nếu cả hai giả thiết trên đều bị bác bỏ thì SDM là phù hợp với dữ liệu hơn. Ngoài ra sử dụng kiểm định LR để kiểm tra các hiệu ứng không gian $H_{0}: \mu_{i}=0$ và các hiệu ứng thời gian $H_{0}: v_{t}=0$. Đối với các mô hình dữ liệu bảng, nó có thể là mô hình hiệu ứng cố định hoặc mô hình hiệu ứng ngẫu nhiên, và khi đó sử dụng kiểm định Hausman để chọn mô hình thích hợp.

\section{Nghiên cứu thực nghiệm}

\subsection{Mô hình nghiên cúu thục nghiệm}

Doanh thu du lịch và các yếu tố tác động đến doanh thu du lịch là chủ đề được quan tâm của nhiều nhà khoa học trên thế giới (Ekanayake \& Long, 2012; Khalil, Kakar, \& Waliullah, 2007; Kim, Chen, \& Jang, 2006; Lee \& Chang, 2008; Lee \& Chien, 2008; Mahmoudinia, Soderjani, \& Pourshahabi, 2011; Naude \& Saayman, 2005; Nissan, Galindo, \& Méndez, 2011; Oh, 2005; Popescu, 2016). Tuy nhiên, phần lớn các nghiên cứu được thực hiện tại nước ngoài, và chưa quan tâm đến vấn đề tương tác không gian giữa các quốc gia hoặc các địa phương của quốc gia trong doanh thu về du lịch.

Tại Việt Nam, chủ đề doanh thu du lịch cũng thu hút được sự quan tâm của các nhà khoa học (Nguyen, Nguyen, \& Nguyen, 2014; Shih \& Do, 2016) nhưng lại nghiên cứu về tác động của doanh thu du lịch đến GDP mà chưa xem xét theo chiều ngược lại. 
Trong nghiên cứu này, nhóm tác giả sử dụng mô hình hồi quy không gian để phân tích các yếu tố tác động đến doanh thu du lịch. Dựa trên kết quả tổng quan lý thuyết kết hợp với nghiên cứu định tính (được trình bày chi tiết tại mục phương pháp nghiên cứu) cho thấy: doanh thu du lịch của một địa phương chịu tác động bởi: GDP, Lượng khách du lịch tại một địa phương, số lượng luân chuyển của hành khách, và dân số.

Doanh thu du lịch: được đo bằng tổng doanh thu từ du lịch của một địa phương.

GDP: được đo lường bởi tổng sản phẩm quốc nội tính theo giá hiện hành của 63 tỉnh/thành của Việt Nam. Thêm vào đó, kết quả nghiên cứu của Ekanayake và Long (2012), Nissan và cộng sự (2011), Mahmoudinia và cộng sự (2011), Lee và Chang (2008), Naude và Saayman (2005) đều cho thấy: GDP là yếu tố có tác động đến doanh thu du lịch. Vì vậy, giả thuyết $\mathrm{H} 1$ được đề xuất như sau:

\section{H1: GDP của các tỉnh/thành có tác động đến doanh thu du lịch của các tỉnh/thành (+)}

Lượng khách được đo bởi tổng lượng khách du lịch tại một địa phương bao gồm cả khách du lịch nội địa và khách quốc tế. Kết quả nghiên cứu của Popescu (2016) cho thấy: lượng khách du lịch tại một địa phương là yếu tố tác động đến đến doanh thu du lịch của địa phương đó. Vì vậy, giả thuyết $\mathrm{H} 2$ được đề xuất như sau:

H2: Lượng khách du lịch của các tỉnh/thành có tác động đến doanh thu du lịch của các tỉnh/thành (+)

Dân số: được đo bằng tổng dân số tại một địa phương.

Số lượng luân chuyển của khách du lịch: được đo bằng số lượng km mà mỗi khách du lịch di chuyển của mỗi địa phương.

Thêm vào đó, kết quả nghiên cứu định tính cho thấy: dân số và số lượng luân chuyển hành khách có tác động đến doanh thu du lịch của mỗi địa phương. Vì vậy, giá thuyết $\mathrm{H} 3$, và H4 được đề xuất như sau:

H3: Dân số của các tỉnh/thành có tác động đến doanh thu du lịch của các tỉnh/thành (+)

H4: Số lượng luân chuyển của khách du lịch của các tỉnh/thành có tác động đến doanh thu du lịch của các tỉnh/thành (+)

\subsection{Quy trình nghiên cúu và nguồn dũ liệu}

\section{Về quy trình nghiên cứu}

Trong nghiên cứu này, nhóm tác giả sử dụng phương pháp nghiên cứu định tính kết hợp định lượng. Nghiên cứu định tính được thực hiện thông qua phỏng vấn sâu cùng 09 chuyên gia (03 nhà khoa học, 03 nhà quản lý về du lịch, và 03 giám đốc doanh nghiệp kinh doanh du lịch và lữ hành). Kết quả nghiên cứu cho thấy: (i) Một là, 09/09 chuyên gia đều thống nhất cho rằng: doanh thu du lịch chịu tác động bởi: GDP, lượng khách du lịch; (ii) 05/09 chuyên gia cho rằng: doanh thu du lịch chịu tác động bởi: dân số của mỗi địa phương, và số lượng luân chuyển hành khách. Nghiên cứu định lượng được thực hiện thông qua ứng dụng mô hình kinh tế lượng không gian để kiểm định mô hình và các giả thuyết nghiên cứu. 


\section{Về nguồn dữ liệu}

Nguồn dữ liệu thứ cấp được trích xuất từ niên giám thống kê của 63 tỉnh thành tại Việt Nam từ năm 2013 đến 2017.

\section{Bảng 1}

Cách thức đo lường và nguồn của các biến trong mô hình

\begin{tabular}{|c|c|l|c|l|}
\hline Tên biến & Ký hiệu & \multicolumn{1}{|c|}{ Định nghĩa biến } & $\begin{array}{c}\text { Kỳ } \\
\text { vọng }\end{array}$ & \multicolumn{1}{|c|}{ Nguồn } \\
\hline $\begin{array}{c}\text { Doanh thu du } \\
\text { lịch }\end{array}$ & doanhthu & $\begin{array}{l}\text { Doanh thu du lịch của } 63 \\
\text { tỉnh/thành của Việt Nam }\end{array}$ & $\begin{array}{l}\text { Niêm giám thống kê của } \\
63 \text { tỉnh/thành của Việt } \\
\text { Nam }\end{array}$ \\
\hline GDP & $g d p$ & $\begin{array}{l}\text { Tổng sản phẩm quốc nội của } 63 \\
\text { tỉnh/thành của Việt Nam }\end{array}$ & + & $\begin{array}{l}\text { Niêm giám thống kê của } \\
63 \text { tỉnh/thành của Việt } \\
\text { Nam }\end{array}$ \\
\hline $\begin{array}{c}\text { Lượng khách du } \\
\text { lịch }\end{array}$ & $l k h a c h$ & $\begin{array}{l}\text { Tổng khách du lịch của } 63 \\
\text { tỉnh/thành của Việt Nam }\end{array}$ & $+\begin{array}{l}\text { Niêm giám thống kê của } \\
63 \text { tỉnh/thành của Việt } \\
\text { Nam }\end{array}$ \\
\hline $\begin{array}{c}\text { Số lượng luân } \\
\text { chuyển của } \\
\text { hành khách }\end{array}$ & $l c h k$ & $\begin{array}{l}\text { Số lượng luân chuyển của hành } \\
\text { khách theo từng địa phương của } \\
63 \text { tỉnh/thành của Việt Nam }\end{array}$ & + & $\begin{array}{l}\text { Niêm giám thống kê của } \\
63 \text { tỉnh/thành của Việt } \\
\text { Nam }\end{array}$ \\
\hline Dân số & danso & $\begin{array}{l}\text { Dân số của } 63 \text { tỉnh thành của } \\
\text { Việt Nam }\end{array}$ & $\begin{array}{l}\text { Niêm giám thống kê của } \\
63 \text { tỉnh/thành của Việt } \\
\text { Nam }\end{array}$ \\
\hline
\end{tabular}

Nguồn: Kết quả phân tích dữ liệu của nhóm nghiên cứu

\section{Kết quả nghiên cứu}

\subsection{Kết quả thống kê mô tả các biến quan sát}

Kết quả thống kê mô tả các biến quan sát chưa lấy logarit được trình bày như Bảng 2 .

\section{Bảng 2}

Kết quả thống kê mô tả các biến quan sát

\begin{tabular}{|c|c|c|c|c|c|}
\hline & Indoanhthu & lnlkhach & lngdp & lndanso & lnlchk \\
\hline Mean & 2.892 & 9.041 & 10.759 & 7.086 & 6.835 \\
\hline Median & 2.907 & 9.002 & 10.677 & 7.090 & 6.828 \\
\hline Maximum & 9.857 & 13.527 & 13.968 & 9.041 & 9.924 \\
\hline Minimum & -2.303 & 5.930 & 8.753 & 5.721 & 3.190 \\
\hline 1st Qu & 1.458 & 7.845 & 10.201 & 6.729 & 6.208 \\
\hline 3rdQu & 4.106 & 9.996 & 11.101 & 7.388 & 7.412 \\
\hline Só́ quan sát & 315 & 315 & 315 & 315 & 315 \\
\hline
\end{tabular}

Nguồn: Kết quả xử lý dữ liệu điều tra 


\subsection{Kiểm định tương quan không gian về tăng trưởng doanh thu du lịch}

Kết quả kiểm định về tương quan không gian theo hệ số Moran's I của biến $\log ($ doanhthu) được trình bày trong Bảng 3 như sau:

\section{Bảng 3}

Kết quả kiểm định về tác động không gian của hệ số Moran’s I

\begin{tabular}{|c|c|c|c|}
\hline Năm & Moran's I & Kỳ vọng & p-value \\
\hline 2013 & 0.193155265 & -0.016129032 & 0.008975 \\
\hline 2014 & 0.190597035 & -0.016129032 & 0.00949 \\
\hline 2015 & 0.15127290 & -0.01612903 & 0.02831 \\
\hline 2016 & 0.160077951 & -0.016129032 & 0.02252 \\
\hline 2017 & 0.139395690 & -0.016129032 & 0.03838 \\
\hline
\end{tabular}

Nguồn: Kết quả xử lý dữ liệu điều tra

Kết quả trên cho thấy các hệ số Moran's I dương và có ý nghĩa thống kê ở mức $1 \%$ và $5 \%$, tức là có tương quan không gian cao giữa các tỉnh/thành Việt Nam và có tương quan cùng chiều về tăng trưởng doanh thu du lịch. Tuy nhiên kết quả này chưa cho biết cụ thể địa phương nào nằm trong cụm không gian có giá trị cao hoặc nằm trong cụm giá trị thấp. Để xem xét điều này có thể thực hiện và xem xét chỉ số Moran $\mathrm{G}^{*}$ của biến $\log (\mathrm{doanh}$ thu) của các tỉnh/thành.

Đồ thị phân tán của Moran's I của log(doanhthu) các tỉnh/thành từ 2013 đến 2017 được trình bày như Hình 3 cho thấy: tất cả các tỉnh đều được biểu thị trong bốn góc phần tư khác nhau, và mỗi tỉnh có thể được phân thành các loại: cao-cao $(\mathrm{H}-\mathrm{H})$, thấp-cao $(\mathrm{L}-\mathrm{H})$, thấp-thấp (L-L) và cao-thấp $(\mathrm{H}-\mathrm{L})$, tương ứng với góc phần tư thứ nhất, thứ hai, thứ ba và thứ tư trong biểu đồ phân tán tương ứng. 

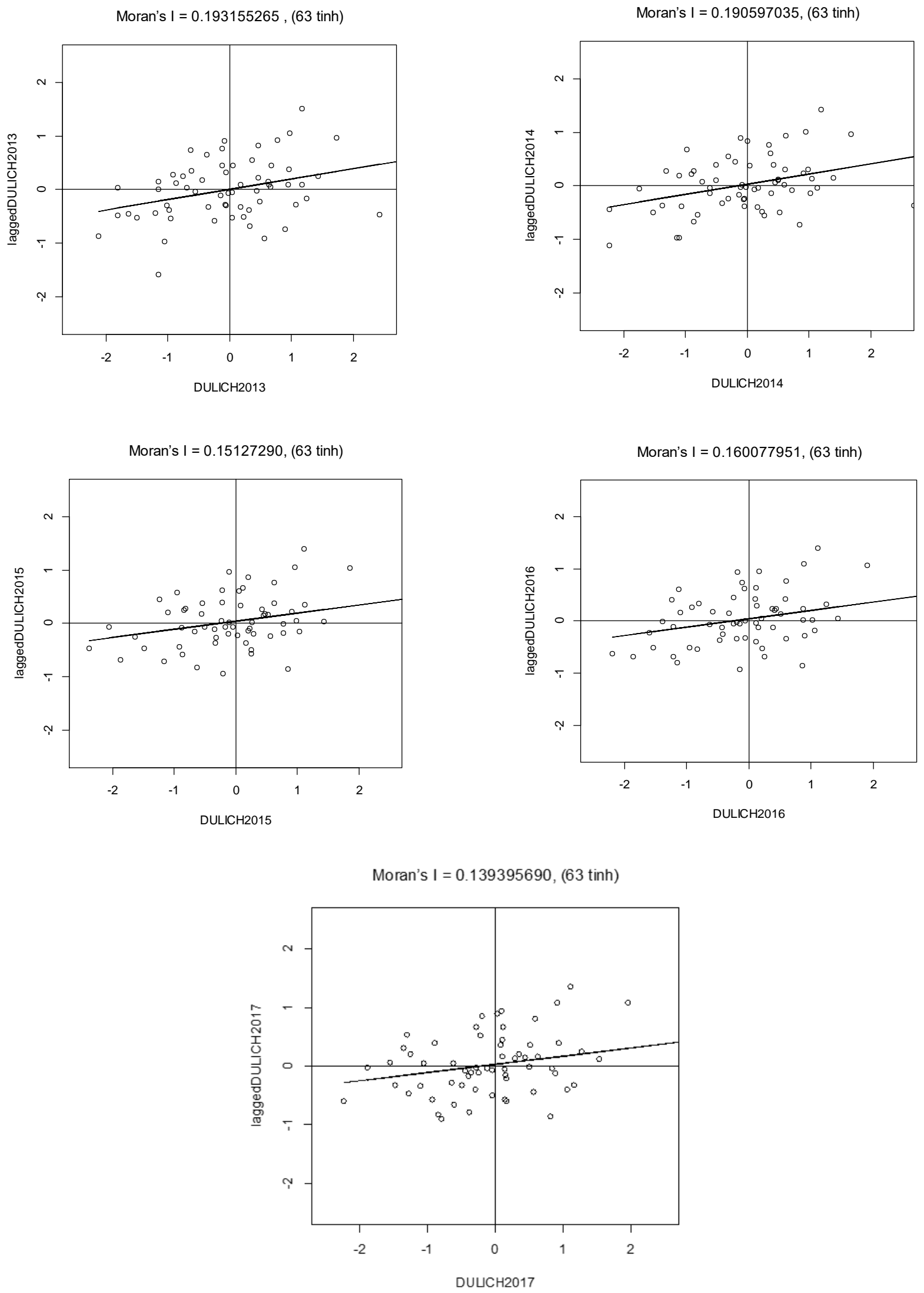

Hình 3. Đồ thị phân tán hệ số Moran’s I của $\log ($ doanhthu) các tỉnh/thành 
Trong bài viết này chỉ nêu ra các tỉnh/thành thuộc cụm giá trị cao (hot spots), và cụm giá trị thấp (cold spots):

\section{Cụm giá trị cao theo từng năm: bao gồm các địa phương}

2013: Huế, Đà Nẵng, Tây Ninh, Đồng Nai, Bà Rịa-Vũng Tàu, TP. HCM, Long An.

2014: Huế, Đà Nẵng, Tây Ninh, Đồng Nai, Bà Rịa-Vũng Tàu, TP. HCM, Long An.

2015: Huế, Đà Nẵng, Đồng Nai, Bà Rịa-Vũng Tàu, TP. HCM, Long An.

2016: Huế, Đà Nẵng, Đồng Nai, Bà Rịa-Vũng Tàu, TP. HCM, Long An.

2017: Huế, Đà Nẵng, Tây Ninh, Đồng Nai, Bà Rịa-Vũng Tàu, TP. HCM, Long An.

\section{Cụm giá trị thấp theo từng năm: bao gồm các địa phương}

2013: Bắc Kạn, Tuyên Quang, Yên Bái, Phú Thọ, Điện Biên, Lai Châu, Sơn La, Thanh Hóa, Đắc Nông.

2014: Cao Bằng, Bắc Kạn, Tuyên Quang, Yên Bái, Điện Biên, Lai Châu, Sơn La, Sóc Trăng. 2015: Cao Bằng, Bắc Kạn, Tuyên Quang, Điện Biên, Lai Châu, Sơn La, Sóc Trăng.

2016: Cao Bằng, Bắc Kạn, Tuyên Quang, Điện Biên, Lai Châu, Sơn La, Sóc Trăng, Bạc Liêu. 2017: Cao Bằng, Bắc Kạn, Tuyên Quang, Điện Biên, Lai Châu, Sơn La, Sóc Trăng.

\subsection{Kiểm định lụa chọn mô hình}

Mô hình phi không gian xác định có dạng như sau:

lndoanhthu $u_{i t}=\beta_{1}$ lnlkhach $_{i t} \beta_{2} \operatorname{lng}_{\text {ln }} p_{i t}+\beta_{3}$ lndanso $_{i t}+\beta_{4} \operatorname{lnlch}_{i t}+\mu_{i}+v_{t}+\varepsilon_{i t}$

Kết quả ước lượng các mô hình không có tác động không gian được trình bày như Bảng 4 .

\section{Bảng 4}

Kết quả ước lượng các mô hình không có tác động không gian

\begin{tabular}{|c|c|c|c|c|}
\hline Tên biến & PooledOLS & Spatial FE & Time-period FE & Two-ways FE \\
\hline lnlkhach & $\begin{array}{l}0.428651 * * * \\
(4.87)\end{array}$ & $\begin{array}{l}0.48946 * * * \\
(4.82)\end{array}$ & $\begin{array}{l}0.395261 * * * \\
(4.29)\end{array}$ & $\begin{array}{l}0.45425 * * * \\
(4.24)\end{array}$ \\
\hline $\operatorname{lng} d p$ & $\begin{array}{l}0.415548 * * * \\
(2.89)\end{array}$ & $\begin{array}{l}0.28851 * \\
(1.80)\end{array}$ & $\begin{array}{l}0.368762 * * \\
(2.47)\end{array}$ & $\begin{array}{l}0.21622 \\
(1.30)\end{array}$ \\
\hline lndanso & $\begin{array}{l}0.277123 * * * \\
(0.84)\end{array}$ & $\begin{array}{l}0.15846 \\
(0.42)\end{array}$ & $\begin{array}{l}0.456507 \\
(1.37)\end{array}$ & $\begin{array}{l}0.3797 \\
(1.01)\end{array}$ \\
\hline lnlchk & $\begin{array}{l}0.538124 * * * \\
(3.94)\end{array}$ & $\begin{array}{l}0.58314 * * * \\
(3.86)\end{array}$ & $\begin{array}{l}0.437823 * * * \\
(2.81)\end{array}$ & $\begin{array}{l}0.46128 * * * \\
(2.63)\end{array}$ \\
\hline Hệ số chặn & $\begin{array}{l}-11.096744 * * * \\
(-8.4626)\end{array}$ & & & \\
\hline$R^{2}$ & 0.54232 & 0.55259 & 0.57502 & 0.57618 \\
\hline $\log L$ & -570.6874 & -544.2592 & -575.539 & -532.7174 \\
\hline LM spatial lag & $5.108 * *$ & $4.6428 * *$ & $6.9254 * * *$ & $6.87 * * *$ \\
\hline LM spatial error & $24.802 * * *$ & $16.717 * * *$ & $21.638 * * *$ & $17.214 * * *$ \\
\hline robust LM spatial lag & $11.122 * * *$ & $4.4009 * *$ & $7.3562 * * *$ & $3.5682 *$ \\
\hline robust LM spatial error & $30.816^{* * *}$ & $16.475 * * *$ & $22.069 * * *$ & $13.912 * * *$ \\
\hline kiểm định Hausman & \multicolumn{4}{|c|}{14.251 với giá trị $\mathrm{p}-$ value $=0.0026$} \\
\hline
\end{tabular}

Nguồn: Kết quả xử lý dữ liệu điều tra 


\section{Kết quả các kiểm định}

Kiểm định LR về tác động cố định không gian có thống kê kiểm định LR là 85.6432 với giá trị $\mathrm{p}$-value $=0.025$, và kiểm định $\mathrm{LR}$ về tác động cố định thời gian có thống kê kiểm định là 23.08 với giá trị $\mathrm{p}$-value $=0.000$. Do đó các hiệu ứng cố định không gian và hiệu ứng cố định thời gian có ý nghĩa thống kê. Vì vậy, trong bài viết này sẽ sử dụng mô hình với các hiệu ứng cố định không gian và thời gian.

Kiểm định LM được sử dụng để xác định xem mô hình kinh tế lượng không gian hay mô hình ước lượng OLS truyền thống là phù hợp.

Kết quả kiểm định LM cho SLM có thống kê kiểm định $\mathrm{LM}=6.87$ với giá trị $\mathrm{p}$-value $=0.008765$, và kiểm định robust $\mathrm{LM}$ có thống kê kiểm định là 3.5682 với giá trị $\mathrm{p}$-value $=$ 0.05889 có ý nghĩa thống kê mức $6 \%$. Đối với SEM có thống kê kiểm định $\mathrm{LM}=17.214$ với giá trị $\mathrm{p}$-value $=0.000$, và kiểm định robust $\mathrm{LM}$ có thống kê kiểm định là $13.912, \mathrm{p}$-value $=$ 0.000 có ý nghĩa mức $1 \%$ cho thấy rằng các mô hình kinh tế lượng không gian là phù hợp hơn ước lượng bằng OLS truyền thống. Ngoài ra kiểm định Hausman có ý nghĩa thống kê mức $1 \%$ điều này chỉ ra rằng mô hình các tác động cố định là phù hợp (Kiểm định Hausman cho dữ liệu bảng không gian cũng cho kết quả tương tự).

Kết quả ước lượng bằng mô hình hồi quy không gian với dữ liệu bảng Để xem xét SDM có thể được đơn giản hóa thành SLM hoặc SEM hay không.

Mô hình độ trễ không gian SLM như sau:

$\log ($ doanhthu $)=\rho W \log ($ doanhthu $)+\beta_{1} \log ($ lkhach $)+\beta_{2} \log ($ gdp $)+\beta_{3} \log ($ danso $)+$

$$
\beta_{4} \log (\mathrm{lch})+\mu+v+u
$$

Mô hình sai số không gian (SEM):

$$
\begin{aligned}
\log (\text { doanhthu })= & \rho W \log (\text { doanhthu })+\beta_{1} \log (\text { lkhach })+\beta_{2} \log (\text { gdp })+\beta_{3} \log (\text { danso })+ \\
& \beta_{4} \log (\text { lchk })+\mu+v+\varepsilon \\
\varepsilon= & \lambda W \varepsilon+u
\end{aligned}
$$

Mô hình Durbin không gian SDM:

$$
\begin{aligned}
\log (\text { doanhthu })= & \rho W \log (\text { doanhthu })+\beta_{1} \log (\text { lkhach })+\beta_{2} \log (\text { gdp })+\beta_{3} \log (\text { danso })+ \\
& \beta_{4} \log (\text { lchk })+\theta_{1} W \log (\text { lkhach })+\theta_{2} W \log (\text { gdp })+\theta_{3} W \log (\text { danso })+ \\
& \theta_{4} W \log (\text { lchk })+\mu+v+u
\end{aligned}
$$

Kết quả ước lượng các mô hình không gian được trình bày như Bảng 5 .

\section{Bảng 5}

Kết quả ước lượng các mô hình không gian như sau:

\begin{tabular}{|l|c|c|c|}
\hline & SEM & SLM & SDM \\
\hline \multirow{2}{*}{ lnlkhach } & $0.309648 * * *$ & $0.324619 * * *$ & $0.268459 * * *$ \\
& $(3.25)$ & $(3.96)$ & $(2.70)$ \\
\hline \multirow{2}{*}{$\ln g d p$} & 0.073513 & -0.010533 & -0.026764 \\
& $(0.47)$ & $(-0.08)$ & $(-0.16)$ \\
\hline
\end{tabular}




\begin{tabular}{|c|c|c|c|}
\hline & SEM & SLM & SDM \\
\hline lndanso & $\begin{array}{c}0.758158^{* * *} \\
(2.07)\end{array}$ & $\begin{array}{c}-0.010533^{* *} \\
(1.99)\end{array}$ & $\begin{array}{c}0.948594 * * \\
(2.39)\end{array}$ \\
\hline $\operatorname{lnlchk}$ & $\begin{array}{c}0.429804 * * * \\
(2.60)\end{array}$ & $\begin{array}{c}0.264351 * * \\
(1.96)\end{array}$ & $\begin{array}{c}0.382244 * * \\
(2.14)\end{array}$ \\
\hline W.lnlkhach & & & $\begin{array}{c}0.014564 \\
(0.11)\end{array}$ \\
\hline$W . \ln g d p$ & & & $\begin{array}{c}0.144952 \\
(0.58) \\
\end{array}$ \\
\hline W.lndanso & & & $\begin{array}{c}-0.910376 \\
(-1.64)\end{array}$ \\
\hline W.lnlchk & & & $\begin{array}{c}-0.186458 \\
(-0.73)\end{array}$ \\
\hline$\lambda$ & $0.56742 * * *$ & & \\
\hline$\rho$ & & $0.463730 * * *$ & $0.549598 * * *$ \\
\hline loglik & -956.6132 & -960.2489 & -954.5609 \\
\hline
\end{tabular}

Nguồn: Kết quả xử lý dữ liệu điều tra

Kiểm định lựa chọn mô hình không gian phù hợp với dữ liệu:

Các giả thiết cần kiểm định $H_{0}: \theta=0$ và $H_{0}: \theta+\rho \beta=0$ để xem xét SDM liệu có thể được đơn giản hóa thành SLM hoặc SEM hay không? Theo kết quả trên: thống kê kiểm định $\mathrm{LR}=4.11$ với giá trị $\mathrm{p}$-value $=0.042<0.05$ cho $\mathrm{SEM}$, và thống kê kiểm định $\mathrm{LR}=11.38$ với giá trị $\mathrm{p}$-value $=0.000<0.01$. Do đó cả hai giả thiết đều bị bác bỏ, vậy SDM là phù hợp với dữ liệu.

Các tác động từ SDM

\begin{tabular}{|l|c|c|c|}
\hline & Trực tiếp & Gián Tiếp & Tổng cộng \\
\hline Lnlkhach & 0.29755569 & 0.29848802 & 0.59604372 \\
\hline lngdp & -0.02966489 & -0.02975783 & -0.05942272 \\
\hline lndanso & 1.05140658 & 1.05470093 & 2.10610751 \\
\hline lnlchk & 0.42367298 & 0.42500047 & 0.84867345 \\
\hline W.lnlkhach & 0.01614294 & 0.01619352 & 0.03233646 \\
\hline W.lngdp & 0.16066189 & 0.16116529 & 0.32182719 \\
\hline W.lndanso & -1.00904568 & -1.01220731 & -2.02125299 \\
\hline W.lnglchk & -0.20666679 & -0.20731434 & -0.41398113 \\
\hline
\end{tabular}

\subsection{Thảo luận kết quả nghiên cúu}

Thông qua tác động trực tiếp, gián tiếp và tổng tác động, mức độ tăng trưởng doanh thu ở các tỉnh/thành của Việt Nam không chỉ phụ thuộc vào yếu tố từ chính địa phương đó mà còn phụ thuộc vào những địa phương lân cận.

\section{Về tác động trụ̣c tiếp}

Xem các yếu tố từ mỗi địa phương sẽ tác động như thế nào đến mức độ tăng trưởng doanh thu hoạt động du lịch của chính nó. Mức độ tăng trưởng doanh thu của một địa phương 
sẽ phụ thuộc vào các yếu tố của ngành du lịch của địa phương đó. Một địa phương có hoạt động du lịch mạnh, sẽ có nhiều khả năng hỗ trợ cho việc tăng trưởng doanh thu mang lại nhiều lợi nhuận hơn cho địa phương. Cụ thể, trong điều kiện các yếu tố khác không đổi khi lượng khách của tỉnh/thành tăng lên $1 \%$ thì tác động trực tiếp đến quy mô tăng trưởng doanh thu của tỉnh tăng khoảng $0.30 \%$, nhưng do tác động phản hồi của mức tác động này, nên tác động trực tiếp của lượng khách đến doanh thu trong tỉnh chỉ tăng trung bình $0.27 \%$. Tương tự khi khối lượng luân chuyển hành khách của các địa phương tăng $1 \%$ thì quy mô doanh thu trong tỉnh tăng trung bình khoảng $0.38 \%$ và khi dân số tỉnh tăng $1 \%$ thì doanh thu tăng trung bình $0.95 \%$ khi các yếu tố khác không đổi. Yếu tố về GDP của tỉnh không có ý nghĩa thổng kê, có thể xem GDP không tác động đến tăng trưởng doanh thu hoặc do dữ liệu hoặc ma trận trọng số không gian chưa phù hợp.

\section{Về tác động gián tiếp}

Tác động gián tiếp (các hiệu ứng lan tỏa không gian) có thể được xem như tác động của một yếu tố của các địa phương lân cận lên quy mô tăng trưởng doanh thu một địa phương cụ thể. Nghĩa là lượng khách, dân số và khối lượng luân chuyển hành khách của các địa phương lân cận có tác động dương lên quy mô tăng trưởng doanh thu của một địa phương cụ thể.

Cụ thể, với các yếu tố khác không đổi khi lượng khách của các tỉnh lân cận tăng lên $1 \%$ thì tác động gián tiếp đến quy mô tăng trưởng doanh thu của địa phương cụ thể tăng khoảng $0.29 \%$.

Tương tự với các yếu tố khác không đổi khi dân số, khối lượng luân chuyển hành khách của các tỉnh lân cận tăng $1 \%$ thì tác động gián tiếp đến quy mô tăng trưởng doanh thu của địa phương cụ thể tăng khoảng $1.05 \%$ và $0.43 \%$ tương ứng.

\section{Về tổng tác động}

Tổng tác động là tổng của tác động trực tiếp và tác động gián tiếp. Tổng tác động được giải thích là sự thay đổi một yếu tố nào đó trong một địa phương sẽ tác động lên việc tăng trưởng doanh thu của chính địa phương đó và các địa phương lân cận.

\section{Kết luận và kiến nghị}

Mặc dù đã có rất nhiều nghiên cứu trong và ngoài nước về quy mô tăng trưởng doanh thu của hoạt động du lịch Việt Nam nói chung và của các tỉnh/thành của Việt Nam nói riêng, nhưng vẫn chưa có nghiên cứu nào tại Việt Nam xem xét sự tương quan không gian về tăng trưởng doanh thu giữa các tỉnh/thành. Bài viết đã cung cấp thêm một hướng nghiên cứu mới về tăng trưởng doanh thu du lịch của các tỉnh/thành tại Việt Nam thông qua mô hình hồi quy không gian. Kết quả nghiên cứu là một bằng chứng thực nghiệm cho thấy có sự tương quan giữa các tỉnh gần nhau của mức độ tăng trưởng doanh thu, các địa phương gần nhau có tương tác không gian nhiều hơn các địa phương ở xa hơn về mặt địa lý. Kết quả nghiên cứu cũng cho thấy: lượng khách, dân số và khối lượng luân chuyển hành khách có sự tương tác về mặt không gian giữa các tỉnh/thành lân cận. Chính vì vậy, các địa phương lân cận về mặt địa lý cần có các chính sách liên kết với nhau thành vùng trong việc đẩy mạnh các hoạt động của địa phương nhằm thúc đẩy tăng trưởng doanh thu của ngành du lịch.

Tuy nhiên, đề tài cũng có một số hạn chế nhất định: một là, bài viết chỉ đưa vào mô hình nghiên cứu một loại ma trận trọng số không gian là ma trận tiếp giáp bậc một, do đó để kết quả có thể phù hợp hơn cần sự so sánh mô hình hồi quy với nhiều loại ma trận trọng số không gian 
khác nhau nhằm chọn lựa được mô hình có ma trận trọng số không gian phù hợp nhất với dữ liệu; hai là, đề tài chỉ đưa vào mô hình chỉ có 4 biến độc lập nhằm giới thiệu sự tương tác không gian, trong các nghiên cứu tiếp theo có thể xem xét thêm các yếu tố khác có thể tác động đến mức độ tăng trưởng doanh thu du lịch như: cơ sở hạ tầng, chất lượng sản phẩm du lịch, số lượng khách sạn, resort, nhà nghỉ tại mỗi tỉnh.

\section{Tài liệu tham khảo}

Anselin, L. (1988). Spatial econometrics: Methods and models. Dordrecht, BO: Kluwer Academic Publishers.

Baltagi, B. H., \& Liu, L. (2008). Testing for random effects and spatial lag dependence in panel data models. Statistics and Probability Letters, 78, 3304-3306.

Chou, K. H., Chen, C. H., \& Mai, C. C. (2015). Factors influencing China's exports with a spatial econometric model. The International Trade Journal, 29(3), 191-211.

Cliff, A. D., \& Ord, J. K. (1981). Spatial processes: Models and applications. London, UK: Pion Limited.

Coughlin, C. C., \& Segev, E. (2000). Foreign direct investment in China: A spatial econometric study. The World Economy, 23, 1-23.

Ekanayake, E. M., \& Long, A. E. (2012). Tourism development and economic growth in developing countries. The International Journal of Business and Finance Research, 6(1), 51-63.

Elhorst, J. P. (2003). Specification and estimation of spatial panel data models. International Regional Science Review, 26(3), 244-268.

Elhorst, J. P. (2014). Spatial panel data models. In Spatial econometrics (pp. 37-93). Heidelberg, Germany: Springer.

Florax, R. J., Folmer, H., \& Rey, S. J. (2006). A comment on specification searches in spatial econometrics: The relevance of Hendry's methodology: A reply. Regional Science and Urban Economics, 36, 300-308.

Khalil, S., Kakar, M., \& Waliullah (2007). Role of tourism in economic growth: Empirical evidence from Pakistan economy. The Pakistan Development Review, 46(4), 985-995.

Kim, H., Chen, M., \& Jang, S. (2006). Tourism expansion and economic development: The case of Taiwan. Tourism Management, 27, 925-933.

Lee, C.-C., \& Chang, C.-P. (2008). Tourism development and economic growth: A closer look at panels. Tourism Management, 29, 180-192.

Lee, C.-C., \& Chien, M.-S. (2008). Structural breaks, tourism development, and economic growth: Evidence from Taiwan. Mathematics and Computers in Simulation, 77, 358-368.

Lee, L., \& Yu, J. (2010). Estimation of spatial autoregressive panel data models with fixed effects. Journal of Econometrics, 154(2), 165-185. 
LeSage, J., \& Pace, R. K. (2009). Introduction to spatial econometrics. Boca Raton, FL: Chapman \& Hall/CRC.

Mahmoudinia, D., Soderjani, E. S., \& Pourshahabi, F. (2011). Economic growth, tourism receipts and exchange rate in MENA zone: Using panel causality technique. Iranian Economic Review, 15(29), 130-146.

Moran, P. A. P. (1950). Notes on continuous stochastic phenomena. Biometrika, 37(1), 17-23.

Mur, J., \& Angulo, A. (2009). Model selection strategies in a spatial setting: Some additional results. Regional Science and Urban Economics, 39, 200-213.

Naude, W. A., \& Saayman, A. (2005). Determinants of tourist arrivals in Africa: A panel data regression analysis. Tourism Economics, 11(3), 365-391.

Nguyen, H. M. T., Nguyen, H. C. D., \& Nguyen, T. D. (2014). Research note: Empirical assessment of the tourism-led growth hypothesis: The case of Vietnam. Tourism Economics, 20, 885-892.

Nissan, E., Galindo, M. A., \& Méndez, M. T. (2011). Relationship between tourism and economic growth. The Service Industries Journal, 31(10), 1567-1572.

Oh, C. (2005). The contribution of tourism development to economic growth in the Korean economy. Tourism Management, 26, 39-44.

Ord, J. K., \& Getis, A. (1995). Local spatial autocorrelation statistics: Distributional issues and an application. Geographical Analysis, 27, 286-306.

Popescu, A. (2016). The correlation between international tourist arrivals and tourism receipts - A key factor of tourism efficiency. Scientific Papers Series Management, Economic Engineering in Agriculture and Rural Development, 16(4), 299-306.

Shih, W., \& Do, N. T. H. (2016). Impact of tourism on long-run economic growth of Vietnam. Modern Economy, 7, 371-376.

Tổng Cục Thống Kê. (2018). Niên giám thống kê của 63 tỉnh/thành của Việt Nam năm 2017 [Statistical Yearbook of 63 provinces / cities of Vietnam in 2017]. Ho Chi Minh, Vietnam: NXB Thống kê.

Tổng Cục Thống Kê. (2019). Niên giám thống kê Việt Nam năm 2018 [Statistical Yearbook of Vietnam 2018] . Ho Chi Minh, Vietnam: NXB Thống kê.

Vega, S. H., \& Elhorst, J. P. (2015). The SLX model. Journal of Regional Science, 55(3), 339363. 